\title{
Shape and topology optimization of a permanent-magnet machine under uncertainties
}

\author{
Piotr Putek ${ }^{1,2^{*}}$ (D), Roland Pulch², Andreas Bartel ${ }^{1}$, E Jan W ter Maten ${ }^{1}$, Michael Günther ${ }^{1}$ and Konstanty \\ M Gawrylczyk ${ }^{3}$
}

"Correspondence:
putek@math.uni-wuppertal.de
${ }^{1}$ Chair of Applied Mathematics and
Numerical Analysis, Bergische
Universität Wuppertal,
Gaußstraße 20, Wuppertal, 42119,
Germany
${ }^{2}$ Institute for Mathematics and
Computer Science,
Ernst-Moritz-Arndt-Universität
Greifswald,
Walther-Rathenau-Straße 47,
Greifswald, 17489, Germany
Full list of author information is
available at the end of the article

\begin{abstract}
Our ultimate goal is a topology optimization for a permanent-magnet (PM) machine, while including material uncertainties. The uncertainties in the output data are, e.g., due to measurement errors in the non-/linear material laws. In the resulting stochastic forward problem, these uncertainties are stochastically modeled by random fields. The solution of the underlying PDE, which describes magnetostatics, is represented using the generalized polynomial chaos expansion. As crucial ingredient we exploit the stochastic collocation method (SCM). Eventually, this leads to a random-dependent bi-objective cost functional, which is comprised of the expectation and the variance. Subject to the optimization of the PM machine are the shapes of the rotor poles, which are described by zero-level sets. Thus, the optimization will be done by redistributing the iron and magnet material over the design domain, which allows to attain an innovative low cogging torque design of an electric machine. For this purpose, the gradient directions are evaluated by using the continuous design sensitivity analysis in conjunction with the SCM. In the end, our numerical result for the optimization of a two-dimensional model demonstrates that the proposed approach is robust and effective.
\end{abstract}

Keywords: robust low cogging torque design; topology and shape optimization; random partial differential equation; stochastic collocation method; level set method; continuous design sensitivity analysis; weighted average method; trade-off method

\section{算 Springer}

\section{Introduction}

Due to high performance, high efficiency and high power density, permanent-magnet (PM) machines are becoming more and more popular [1-3]. Consequently, these devices are currently broadly used in applications as robotics, hybrid vehicles, computer peripherals and so on, see, e.g., [4-8]. However, the PM machines suffer by construction from a considerable level of mechanical vibration and acoustic noise. More precisely, the interaction of the air-gap harmonics (stator slot driven) and the magnetomotive force harmonics (magnet driven) produces a high cogging torque (CT). On the other hand, the torque ripple is primarily provoked by the CT and higher harmonics of back-electromotive force (EMF). Also the magnetic saturation in the stator and the rotor cores [9] as well as the

(c) Putek et al. 2016. This article is distributed under the terms of the Creative Commons Attribution 4.0 International License (http://creativecommons.org/licenses/by/4.0/), which permits unrestricted use, distribution, and reproduction in any medium, provided you give appropriate credit to the original author(s) and the source, provide a link to the Creative Commons license, and indicate if changes were made. 
controller-induced parasitic torque ripples [10] might further disturb the electromagnetic torque [11].

From this perspective, especially the mitigation of the torque fluctuations is a key issue for the design of a PM machine because its result may simultaneously affect the machine performance. Especially, in the context of the deterministic/stochastic topology optimization of a PM machine, the consideration of more than one competing objective into a cost functional seems to be still a challenging problem $[3,12]$. More specifically, when a multi-objective approach is involved in the designing process, a certain trade-off between conflicting criteria needs to be fulfilled. For this reason, often a Pareto front technique is accepted as an alternative. Furthermore, under assumption that the Pareto front is convex, it can be approximated by the weighted average method (WAM) or the $\epsilon$-method [13-15]. However, in practice it is rather hard to verify this assumption, especially when nonlinear problems are considered. In such a situation, objective functions are approximated numerically, whereas a genetic or ant colony-based algorithm is recommended for the identification of the global front Pareto $[3,16]$. It should be also noticed that in some cases, when a periodic functional may be applied, e.g., for a lumped model of electric machine [17] or the periodicity of objectives is a result of the geometrical structure of electric machines [12], it is also possible to prove the convexity in a rigorous mathematical way.

Various methods for suppressing the CT have been proposed in the literature. For example in [18] the authors employed the auxiliary slots for this purpose. Some solutions apply an appropriately chosen combination of slot/pole number [19] or the optimized ratio of pole arc to pole pinch [9] in order to reduce the CT. Other efficient methods for mitigating the CT involve shaping the rotor magnets and/or stator teeth [20] including redistribution of a PM and iron material within the domain of interest using topological methods, as proposed in [6, 8, 21-24]. Moreover, the statistics-based approach such as the Taguchi method [25], or its generalization called the regression-based surface response methods, are proposed in $[11,26]$, especially in industrial applications to reduce the noise to signal ratio. To this last group, also techniques based on the perturbation method for calculating the first and the second derivative may be included. Based on the sensitivity information they intend to estimate deterministically the impact of the input variability on output characteristics and in consequence on the result of optimization [27]. On the one hand, this 'deterministic' estimation of uncertainties is limits to the range of the perturbation $|\delta|=6-8 \%$, see, e.g., [27-29]. On the other hand, the load related to the calculation of the second derivative might be really large, especially when a cost functional involves a standard deviation. Clearly, the advantage of this approach is the immediate availability of a gradient which allows to carry out the strategy, whose aim is to reduce the influence of the deterministic uncertainty onto the optimization result [28]. More recently, the efficient approach, which benefits from both the perturbation technique and the stochastic-based method has been developed [29] to estimate the statistical moments.

In many engineering applications, a physical model is affected by a relatively large amount of uncertainty in the input data including, e.g., the model coefficients, the excitation term, initial/boundary conditions or the geometry of a model. For instance, in the case of an electric machine, the source of the uncertainty has been schematically shown in Figure 1. In this respect, in order to develop a reliable numerical simulation, it is necessary to include uncertainty quantification due to the variations of input data [30]. This differs significantly from the deterministic approach. Depending on the available data, the 


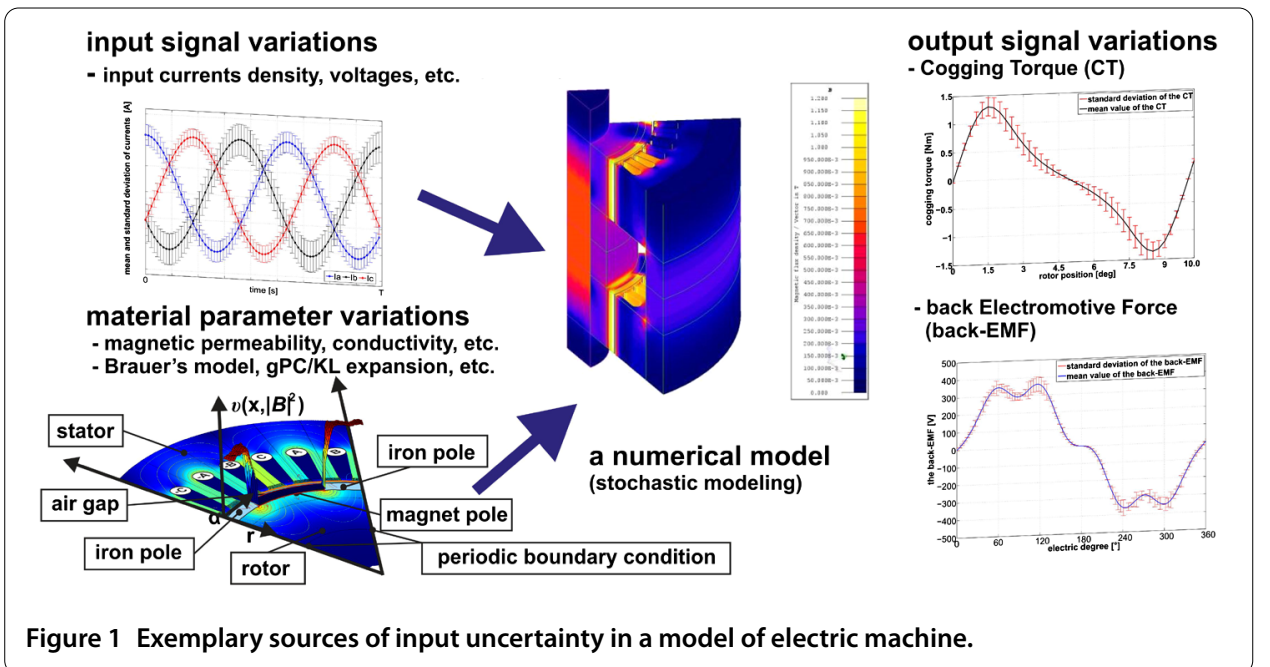

uncertainty can be represented in the mathematical model using the worse-case scenario analysis, evidence theory, fuzzy set theory and probabilistic framework, see, e.g., [31] and references therein. For this reason, in this paper we deal with the non-intrusive approach, namely a type of the spectral collocation method [32], combined with the generalized polynomial chaos [33], to investigate the propagation of the uncertainty through a model of electric machine. Additionally, this technique allows to straightforwardly include the surface response model into the optimization flow [34-36].

The topology is a major contributor to the electromagnetic torque fluctuations. Therefore in this paper, we address the topology optimization of a PM machine. Since the results of the design procedure are highly influenced by unknown material characteristics [37], these uncertainties have to be taken into account in the course of a robust optimization. Thus the soft ferromagnetic material should be modeled using uncertainty. In particular, the relative permeability/reluctivity of the magnetic material needs an accurate model in order to improve the accuracy of the magnetic flux density of permanent magnets (for certain applications $[38,39])$. Therefore, in our optimization model, we take the reluctivity as uncertain.

In our case of the topology optimization, we have to trace two interfaces between different materials with some assumed variations such as air, iron and PM poles of rotor, the modified multilevel set method (MLSM) has been used [40, 41]. The level set method [42] has found a wide range of applications also in electrical engineering. For instance, it is used to address shape or topology optimization problems [6,22].

The proposed approach is innovative, since stochastic modeling of uncertainties are combined with a topology optimization for minimal electromagnetic torque fluctuations (of the CT) and at the same time allowing for a robust optimization on the basis of a suitable optimization criterion. The current paper is an extended version of [35] with many more details and new results for an analysis of a PM electric machine in on-load state (with excitation currents involved). More precisely, we paid a lot of attention to investigate the impact of the optimization methodology on other machine parameters such as the electromagnetic torque, the torque ripple, the back electromotive force and an analysis of its frequency spectrum as the important source of noise and vibrations. However, it should be noticed that in this paper we focus mainly on a robust low cogging torque design 
under uncertainty which resulted in no-load steady state analysis of stochastic curl-curl equation (a density of excitation currents $J(\mathbf{x})=0$ ). Consequently, the machine analysis in on-load state can be considered here as a post-processing procedure, since the electromagnetic (average) torque itself was not involved in the optimization task. Moreover, we restricted ourselves to the minimization of noise and vibrations caused predominantly by the cogging torque, the torque ripple and the back EMF, however, without coupling a vibro-acoustic modeling as in, e.g., [43] with the curl-curl equation. Instead the air-gap flux density (as equivalence of the back EMF) is considered in the optimization procedure as a second objective. It yields a considerably improvement of the wave form of the back electromotive which, in turn, directly leads to the reduction of noise and vibrations. Furthermore, to deal with a stochastic multi-objective problem, the trade-off method, incorporated in the level set method (LSM) as well as the AWM, involved in a robust functional, have been applied.

The paper is organized as follows: first we describe the PM machine, which we use as test case (Section 2). Then the deterministic model is set up (Section 3). Based on that, the stochastic forward problem is formulated in Section 4. Section 5 describes the optimization problem with the needed objective functions and the constraints. Then we combine topology optimization and uncertainties (Section 6). After a short description of the simulation in Section 7, we discuss numerical results (Section 8).

\section{Test case description}

A design of a PM machine has to provide the shape and placement of magnets, iron poles and air-gaps. These features primarily determine the torque characteristics and thus the proper and efficient functioning of a PM machine. Specifically, we consider as test case an electric controlled permanent magnet excited synchronous machine (ECPSM) [44]. For illustration, Figure 2 provides a partial assembly drawing of such a device and the related main parameters for the magnetic description are found in Table 1. In fact, the ECPSM rotor consists of two almost identical parts, which just have opposing direction of the PM poles (see Figure 2 'permanent magnet $N$ ' and 'permanent magnet $S$ '). Furthermore, an additional DC control coil is mounted in the axial center of the machine, actually between the laminated stators. Via a DC-chopper, this allows for controlling the effective excitation of the machine. Eventually this results in a field weakening of $1: 4$, which is of particular importance for electric propulsion vehicles [44].
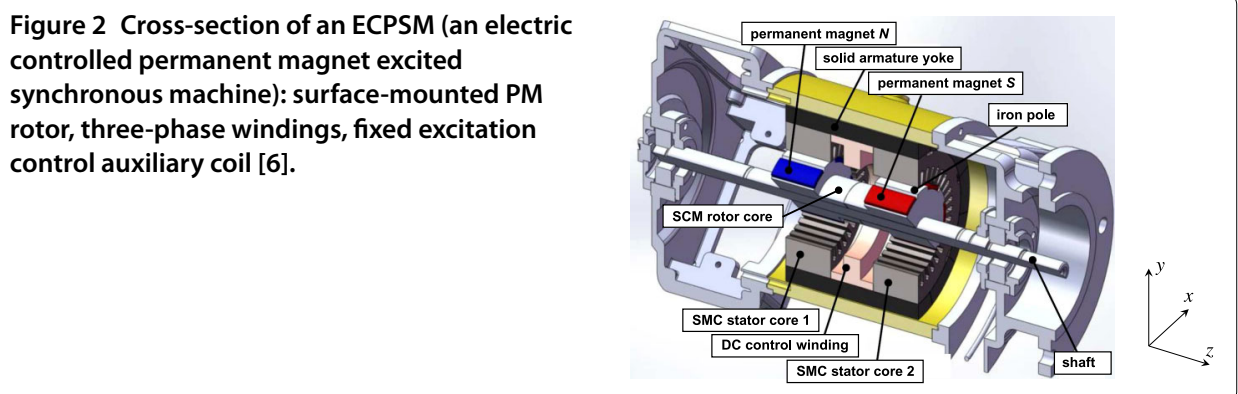
Table 1 Main parameters of the ECPSM (an electric controlled permanent magnet excited synchronous machine) design [6]

\begin{tabular}{lll}
\hline Parameter (unit) & Symbol & Value \\
\hline Pole number & $2 \mathrm{p}$ & 12 \\
Stator outer radius $(\mathrm{mm})$ & $r_{\text {ostat }}$ & 67.50 \\
Stator inner radius $(\mathrm{mm})$ & $r_{\text {istat }}$ & 41.25 \\
One part stator axial length $(\mathrm{mm})$ & $I_{\text {as }}$ & 35.0 \\
Slot opening width $(\mathrm{mm})$ & $W_{\text {oslot }}$ & 4.0 \\
Number of slots & $n s$ & 36 \\
Number of phases & $m$ & 3 \\
Permanent magnet pole & $\mathrm{NdFeB}$ & 12 \\
PM thickness (mm) & $t_{\mathrm{m}}$ & 3.0 \\
Remanent flux density $(\mathrm{T})$ & $B_{\mathrm{r}}$ & 1.2 \\
\hline
\end{tabular}

Figure 3 Sextant domain for mathematical model: stator, rotor and three phases $(A, B, C)$.

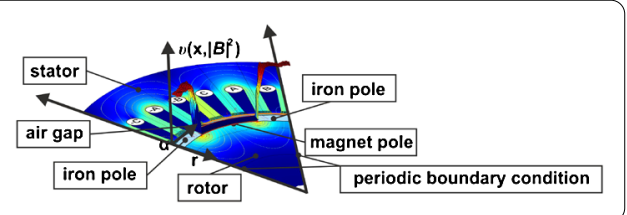

\section{Mathematical model}

In the following, we set up a deterministic model for the PM machine, which is suitable for optimization. This comprises a discussion of the domain, a strong and weak formulation and the objective functions.

\subsection{Field quantity and simulation domain}

The magnetic behavior of the PM machine can be formulated in terms of the unknown magnetic vector potential $\mathbf{A}$ and the so-called curl-curl equation. Here we disregard eddy currents, i.e., we assume $\sigma \frac{\partial \mathbf{A}}{\partial t}=0$, where $\sigma$ denotes the conductivity. Especially for optimization, one needs an efficient computational model. Therefore we reduce the three (spatial-) dimensions of the problem into a simplified 2D FEM model, which provide us with acceptably accurate computation result. We validated this approach in our previous work $[6,41]$ using numerical simulation and experiment in the case of a similar topology. Thus $\mathbf{A}=(0,0, A(x, y))$, which gives us a $2 \mathrm{D}$ problem. Moreover, this setting exhibits rotational symmetry with multiples of six. Hence we restrict the domain to one sextant, see Figure 3.

\subsection{Strong formulation}

Now, in 2D the curl-curl equation for the magnetic vector potential $A=A(x, y)$ becomes the following Poisson equation:

$$
\nabla \cdot\left(v\left(\mathbf{x},|\nabla A(\mathbf{x})|^{2}\right) \nabla A(\mathbf{x})-v_{\mathrm{PM}} \mathbf{M}(\mathbf{x})\right)=J(\mathbf{x}), \quad \mathbf{x} \in \mathrm{D} \subset \mathbb{R}^{2},
$$

where the domain $\mathrm{D}$ denotes the sextant region in our test case. Here $J(\mathbf{x})$ denotes the given current density (at position $\mathbf{x}=(x, y)), \mathbf{M}(\mathbf{x})$ represents the given remanent flux density of the PM, $v$ is the reluctivity and $v_{\mathrm{PM}}$ the reluctivity of the permanent magnets. Moreover, we assume

$$
\mathbf{M}(\mathbf{x}):=b_{r}(\mathbf{x}) \mathbf{T}(\mathbf{x})
$$


where $\mathbf{T}(\mathbf{x})$ describes the radial direction of the remanent flux density and $b_{r}(\mathbf{x})$ denotes a positive and bounded scalar function for the respective magnitude.

This quasi-linear elliptic problem (1) is equipped with periodic boundary conditions on the radii of $\mathrm{D}$ and it is also equipped with a homogeneous Dirichlet condition on the outer arc: $A(\mathbf{x})=0$.

The reluctivity $v$ describes as real parameter the material relation $\mathbf{H}=v\left(\mathbf{x},|\mathbf{B}|^{2}\right) \mathbf{B}$, where $\mathbf{B}=\nabla \times \mathbf{A}$ and $\mathbf{H}$ denote the magnetic flux density and the magnetic field strength, respectively. On the one hand, it depends nonlinearly on $B:=|\mathbf{B}|=|\nabla \times \mathbf{A}|=|\nabla A|$, where $|\cdot|$ denotes the Euclidean norm and we use the $2 \mathrm{D}$ setting $\mathbf{A}=(0,0, A(x, y))$. On the other hand, the reluctivity depends on the respective local material, i.e., it depends on the position $\mathbf{x}$. In our case, our connected domain is composed of iron ( $\mathrm{Fe})$, air, and permanent magnets:

$$
\mathrm{D}=\mathrm{D}_{\mathrm{Fe}} \cup \mathrm{D}_{\text {air }} \cup \mathrm{D}_{\mathrm{PM}}
$$

Thus the reluctivity reads

$$
v\left(\mathbf{x},|\nabla A(\mathbf{x})|^{2}\right)= \begin{cases}v_{\mathrm{Fe}}\left(\mathbf{x},|\nabla A(\mathbf{x})|^{2}\right) & \text { for } \mathbf{x} \in \mathrm{D}_{\mathrm{Fe}}, \\ v_{\text {air }} & \text { for } \mathbf{x} \in \mathrm{D}_{\text {air }}, \\ v_{\mathrm{PM}} & \text { for } \mathbf{x} \in \mathrm{D}_{\mathrm{PM}}\end{cases}
$$

That is, the reluctivity $v$ is discontinuous across material borders and nonlinear in ferromagnetic materials. In the following, we assume that $v_{\text {air }}=v_{0}$ is the vacuum reluctivity and that the electromagnetic material is soft. Moreover, we remark that the nonlinear dependence of $v_{\mathrm{Fe}}$ on $|\nabla A|^{2}$ is given by a spline interpolation of measurement data.

\subsection{Weak form}

We assume $J \in L^{2}\left(\mathrm{D}_{\mathrm{F}}\right)$ and $\mathrm{M} \in\left(L^{2}\left(\mathrm{D}_{\mathrm{F}}\right)\right)^{3}$, where $\mathrm{D}_{\mathrm{F}}:=6 \mathrm{D}$ denotes the full circle. A function $A \in V:=H_{0}^{1}\left(\mathrm{D}_{\mathrm{F}}\right)=\left\{A \in H^{1}\left(\mathrm{D}_{\mathrm{F}}\right):\left.A\right|_{\partial \mathrm{D}_{\mathrm{F}}}=0\right\}$ is a weak solution of the quasi-linear elliptic Dirichlet boundary problem (1) if it holds

$$
\underbrace{\int_{\mathrm{D}_{\mathrm{F}}}\left(v\left(\mathbf{x},|\nabla A|^{2}\right) \nabla \varphi \nabla A\right) \mathrm{d} \mathbf{x}}_{=: a(\varphi, A)}=\underbrace{\int_{\mathrm{D}_{\mathrm{F}}} \varphi J \mathrm{~d} \mathbf{x}}_{=: l_{1}(\varphi, J)}+\underbrace{v_{\mathrm{PM}} \int_{\mathrm{D}_{\mathrm{F}}} \mathbf{M} \cdot \nabla \varphi \mathrm{d} \mathbf{x}}_{=: l_{2}(\varphi, \mathbf{M})}
$$

for all $\varphi \in V$. The symbol $H^{1}\left(\mathrm{D}_{\mathrm{F}}\right)$ denotes the Sobolev space of the real-valued functions on $\mathrm{D}_{\mathrm{F}}$ with square integrable weak gradients. The existence and uniqueness of solution for problem (4) is thoroughly investigated in, e.g., [45].

\section{Stochastic forward problem}

The characteristics of ferromagnetic materials are usually deduced from measurements. In our case, this applies to the reluctivity $v$, which suffers from measurement uncertainties. Now, the result for the electrical machine design is strongly affected by the unknown material characteristics and geometric uncertainties, for example, the air-gap thickness, see in $[28,37]$. The latter effect can be also simulated by changing the material parameter using the level set methodology (see below). This leaves us to include these material uncertainties into the mathematical model and thus into the optimization procedure in 
order to achieve a robust design. Therefore, in our work, the reluctivity becomes a random field, which allows us to quantify these uncertainties. The respective modeling is our next subject.

\subsection{Stochastic modeling of uncertain reluctivities}

We recall the reluctivity $v$ (3), which is material dependent. We have: iron, air and permanent magnet domains. Besides the variations of iron and permanent magnet reluctivities, especially the uncertainty of $v_{\text {air }}$ is crucial from the engineering viewpoint, since it allows us to simulate the variations of the air-gap thickness. For an uncertainty quantification, the reluctivities become random variables on some probability space $(\Omega, \mathscr{F}, \mathbb{P})$ with sample space $\Omega$, sigma-algebra $\mathscr{F}$ and probability measure $\mathbb{P}$. In our stochastic model, we introduce random perturbations within the material parameters. The random field reads as

$$
v(\mathbf{x},|\nabla A|, \boldsymbol{\xi})= \begin{cases}v_{\mathrm{Fe}}\left(\mathbf{x},|A(\mathbf{x})|^{2}\right)\left(1+\delta_{1} \xi_{1}\right) & \text { for } \mathbf{x} \in \mathrm{D}_{\mathrm{Fe}}, \\ v_{\mathrm{air}}\left(1+\delta_{2} \xi_{2}\right) & \text { for } \mathbf{x} \in \mathrm{D}_{\text {air }}, \\ v_{\mathrm{PM}}\left(1+\delta_{3} \xi_{3}\right) & \text { for } \mathbf{x} \in \mathrm{D}_{\mathrm{PM}}\end{cases}
$$

with random vector $\xi=\left(\xi_{1}, \xi_{2}, \xi_{3}\right)$. Therein, the random variables $\xi_{j}(j=1,2,3)$ are assumed to be independent and identically uniformly distributed in the interval $[-1,1]$. Thus, the parameters $\delta_{j}>0(j=1,2,3)$ specify the relative magnitude of the perturbation in the material parameters. For the later numerical simulations, we choose $\delta_{j}=0.1$ for all $j$, which corresponds to perturbations of $10 \%$. Thus we have $\xi: \Omega \rightarrow[-1,1]^{3}=: \Pi$.

For uncertainty quantification, we need the expectation and the variance among other quantities. The expected value of a function $f: \Pi \rightarrow \mathbb{R}$, which depends on the random variables, is defined as

$$
\mathbb{E}[f]:=\int_{\Omega} f(\xi(\omega)) \mathrm{d} \mathbb{P}(\omega)=\int_{\Pi} f(\xi) \rho(\xi) \mathrm{d} \xi
$$

provided that the integral is finite. In our case of uniform random distributions, the joint probability density function is constant: $\rho(\xi)=\frac{1}{8}$. Furthermore, given two functions $f, g$ : $\Pi \rightarrow \mathbb{R}$, the expected value (6) induces an inner product

$$
\langle f, g\rangle:=\mathbb{E}(f g)
$$

on the Hilbert space $L^{2}(\Omega)=\left\{f: \mathbb{E}\left[f^{2}\right]<\infty\right\}$. The variance of a function $f \in L^{2}(\Omega)$ is simply

$$
\operatorname{Var}[f]:=\mathbb{E}\left[f^{2}\right]-\mathbb{E}[f]^{2}
$$

\subsection{Polynomial chaos expansion}

Our uncertainty quantification is based on the concept of the polynomial chaos expansion (PCE). The homogeneous polynomial chaos was introduced in [46] for Gaussian probability distributions. Later this concept was extended to other probability distributions, which resulted in the generalized polynomial chaos, see [33, 47].

Given a function $f \in L^{2}(\Omega)$, the associated PCE reads as

$$
f(\xi)=\sum_{i=0}^{\infty} f_{i} \Psi_{i}(\xi)
$$


The expansion (9) converges in the $L^{2}$-norm of the probability space under certain assumptions $[48,49]$, which are fulfilled for many random distributions such as the Gaussian, uniform and beta distribution. On the one hand, the functions $\Psi_{i}: \Pi \rightarrow \mathbb{R}$ represent a complete orthonormal ${ }^{\mathrm{a}}$ system of basis polynomials. Thus it holds that

$$
\left\langle\Psi_{i}, \Psi_{j}\right\rangle= \begin{cases}0 & \text { for } i \neq j \\ 1 & \text { for } i=j\end{cases}
$$

using the inner product (7). These orthonormal polynomials follow from the choice of the probability distributions in the stochastic modeling. Each traditional probability distribution exhibits its own orthogonal system, see [47]. In our case, the uniform distribution implies the Legendre polynomials as basis. We remark that the multivariate polynomials are just the products of the univariate orthonormal polynomials for each random variable. On the other hand, the coefficients $f_{i}$ in (9) satisfy the relation

$$
f_{i}=\left\langle f, \Psi_{i}\right\rangle \quad \text { for each } i
$$

i.e., they result from the projection of the function $f$ onto the basis polynomials. In our numerical simulation, these coefficients represent the unknowns, which are used to describe the desired solutions of our problem. For traditional random variables, the series (9) is convergent in the norm of $L^{2}(\Omega)$.

We further remark that the PCE (9) includes the information of the expected value (6) as well as the variance (8) of the function $f$ :

$$
\mathbb{E}[f]=f_{0}, \quad \operatorname{Var}[f]=\sum_{i=1}^{\infty} f_{i}^{2}
$$

under the assumption that $\Psi_{0} \equiv 1$ and that the coefficients are given exactly by (10).

To enable a numerical realization, the series (9) has to be truncated at some integer $N_{\max }$. Often all multivariate polynomials up to some total degree are included in the truncated expansion. This truncation causes that the associated variance is just an approximation of the exact variance in (11). Furthermore, the coefficients (10) are typically not given exactly, since they are influenced by numerical errors. If a function $f$ is also space-dependent, then the PCE (9) is considered pointwise for each $\mathbf{x} \in \mathrm{D}$.

\subsection{Stochastic PDE model and PCE approximation}

Now, we return to our PDE problem (1). For the uncertainty quantification, we consider the case of no load state with the excitation density current $J=0$. Consequently, in the Section 5 about the modeling and the optimization problem we will mainly focus on reducing the cogging torque (no-current torque) as an undesirable component for the operation of the ECPSM machine and on improving a wave form of the electromagnetic force when taking the uncertainties into account. Inserting the random field model for the reluctivities (5), we obtain the following stochastic forward problem for our three subdomains $\mathrm{D}=\mathrm{D}_{\text {air }} \cup \mathrm{D}_{\mathrm{Fe}} \cup \mathrm{D}_{\mathrm{PM}}$ :

$$
\begin{cases}\nabla \cdot\left(v_{\mathrm{Fe}}\left(\mathbf{x},|\nabla A(\mathbf{x}, \boldsymbol{\xi})|^{2}, \xi_{1}\right) \nabla A(\mathbf{x}, \boldsymbol{\xi})\right)=0, & \text { in } \mathrm{D}_{\mathrm{Fe}} \\ \nabla \cdot\left(v_{\mathrm{air}}\left(\mathbf{x}, \xi_{2}\right) \nabla A(\mathbf{x}, \boldsymbol{\xi})\right)=0, & \text { in } \mathrm{D}_{\mathrm{air}}, \\ \nabla \cdot\left(v_{\mathrm{PM}}\left(\xi_{3}\right) \nabla A(\mathbf{x}, \boldsymbol{\xi})\right)=\nabla \cdot v_{\mathrm{PM}}\left(\xi_{3}\right) \mathbf{M}(\mathbf{x}), & \text { in } \mathrm{D}_{\mathrm{PM}}\end{cases}
$$


where $A: \mathrm{D} \times \Omega \rightarrow \mathbb{R}, A=A(\mathbf{x}, \boldsymbol{\xi})$ becomes an unknown random field. This random field is approximated by a truncated PCE (9)

$$
A(\mathbf{x}, \boldsymbol{\xi})=\sum_{i=0}^{N_{\max }} a_{i}(\mathbf{x}) \Psi_{i}(\xi)
$$

for some integer $N_{\max }$ and unknown coefficient functions $a_{i}: \mathrm{D} \rightarrow \mathbb{R}$, which are defined by (10) pointwise for $\mathbf{x} \in \mathrm{D}$.

\subsection{Stochastic collocation}

There are mainly two classes of numerical methods for the approximative computation of the coefficient functions $a_{i}$ : stochastic collocation methods (SCM) and stochastic Galerkin techniques, see, e.g., $[33,49]$. We apply the SCM for our problem, since this strategy represents a non-intrusive approach, where the codes for the simulation of the deterministic case can be reused in the stochastic case.

In the SCM, the probabilistic integrals (10) are approximated by a sampling scheme or a multi-dimensional quadrature formula. In fact, any quadrature formula is defined by a set of nodes $\boldsymbol{\xi}^{(k)} \in \Pi$ and a set of weights $w_{k} \in \mathbb{R}$, for $k=1, \ldots, K$. The approximations read as

$$
a_{i}(\mathbf{x})=\left\langle A(\mathbf{x}, \cdot), \Psi_{i}(\cdot)\right\rangle \approx \sum_{k=1}^{K} w_{k} A\left(\mathbf{x}, \boldsymbol{\xi}^{(k)}\right) \Psi_{i}\left(\xi^{(k)}\right)
$$

for $i=0,1, \ldots, N_{\max }$. That is, each term in the sum (14) involves the vector potential $A$ at a particular node in $\Pi$. Thus we have to solve our original PDE problem (1) $K$ times for different realizations of the reluctivity. This computation can be done by separate runs of a numerical method for the deterministic case.

As multi-dimensional quadrature on $[-1,1]^{Q}$, we apply the Stroud formulas with constant weight function, see [50,51]. This type of quadrature methods exhibits an optimality property with respect to the number of required nodes to calculate the integral exactly for all multivariate polynomials up to a total degree $R$. For example, it holds that $K=2 Q$ for $R=3$ and $K=2 Q^{2}+1$ for $R=5$.

Finally, the mean and the standard deviation are obtained from the representation (13) via

$$
\mathbb{E}[A(\mathbf{x}, \cdot)] \approx a_{0}(\mathbf{x}) \quad \text { and } \quad \operatorname{std}[A(\mathbf{x}, \cdot)] \approx \sqrt{\sum_{i=1}^{N}\left|a_{i}(\mathbf{x})\right|^{2}}
$$

using the symbols $a_{i}$ also for the approximation of the exact coefficient functions from (14) for convenience.

\section{Modeling of the optimization problem}

We need to define how the quality of the machine design is measured and the optimization variables as well as their variations.

\subsection{Objective functions}

For the optimization, one has to assess the quality of the design of a PM motor. Here we will have two ingredients, i.e., we set up a bi-objective optimization problem. 
On the one hand, the assessment is based on the cogging torque fluctuations. The cogging torque $T$ can be directly computed from the magnetic field distribution (Maxwell stress tensor method) as a function of the rotor position $\theta[1]$ :

$$
T(\mathbf{x})=v_{\text {air }} \oint_{S} \mathbf{x} \times\left((\mathbf{n} \cdot \mathbf{B}(\mathbf{x})) \mathbf{B}(\mathbf{x})-\frac{|\mathbf{B}(\mathbf{x})|^{2}}{2} \mathbf{n}\right) \mathrm{d} S(\mathbf{x}),
$$

where $\mathbf{n}$ denotes the unit outward normal vector and $S$ a closed surface, which is located in the air-gap and surrounds the rotor. $T$ is mainly determined by the machine's topology.

On the other hand, the root mean square (rms) value of the magnetic field density is employed to assess the quality as second criterion. In fact, this is done only in an approximated way, see [41]: the rms is calculated along a path of length $L$ from location $\boldsymbol{\alpha}_{n}$ to $\boldsymbol{\alpha}_{n+1}$, which lies completely in the air-gap:

$$
\left|B_{\mathrm{r}, \mathrm{rms}}(\mathbf{x})\right|^{2}=\frac{1}{L} \int_{\boldsymbol{\alpha}_{n}}^{\boldsymbol{\alpha}_{n+1}}\left|B_{r}(\mathbf{x})\right|^{2} \mathrm{~d} \mathbf{x} \geq \tau \cdot\left|B_{\mathrm{r}, \mathrm{rms}}{ }^{(0)}\right|^{2} .
$$

Here $\tau$ denotes an assumed level of the magnetic flux density in the air-gap, treated here as the fraction of the value $\left|B_{\mathrm{r}, \mathrm{rms}}{ }^{(0)}\right|^{2}$, which is calculated for the initial configuration using (17).

Alternatively, the back-EMF (electromotive force) can be considered as a second objective in the topology optimization problem [24]. It results from the fact, that the harmonic content of the back-EMF is primarily responsible for the pulsation in the developed electromagnetic torque $[9,52]$. Thus, the square of the back-EMF magnitude is defined as

$$
\left|U_{\text {back }}(\mathbf{x})\right|^{2}:=L_{S} N_{\mathrm{wt}} 2 \pi \omega^{2} \sum_{i=1}^{m} \frac{1}{\left|\mathrm{~S}_{i}\right|} \int_{\mathrm{S}_{i}}|\mathbf{A}(\mathbf{x})|^{2} \mathrm{~d} \mathbf{x},
$$

where $L_{\mathrm{S}}$ refers to the axial length of the stator, $N_{\mathrm{wt}}$ denotes the number of winding turns, $S_{i}$ represents the cross-section area of the windings for every phase, while $m$ specifies the number of phases.

From engineering viewpoint, both objectives, which compete each other, are very important. The first of them as a main component of the torque ripple is responsible for minimizing the noise and vibrations, which are crucial for the low-speed application. While the second function allows for ensuring possibly the bigger value of the flux density calculated in air-gap or, equivalently, the highest value of $U_{\text {back }}$. The spectrum of the latter has also impact on vibrations. As a result, it influences the electromagnetic torque as well. For a solution of the multi-objective problem the $\epsilon$-method [13], incorporated in the LSM scheme has been applied. It means that a second criterion serves as a constraint bounded by some allowable range of parameters $\epsilon$. On the one hand, this method requires some technical information about objectives preferences as well as the convexity of a Pareto front, what for the periodic functions is often fulfilled. On the other hand, the obtained solution might not necessary be globally non-dominated [53] due to the treatment of the $B_{\mathrm{r}}$ objective as the fraction of areas. Therefore, in order to find a non-dominated globally solution a Pareto front technique need to be applied for a robust optimization. 
Figure 4 Distribution of the signed distance function [35]. Here the shapes of rotors poles (the blue shape with black lines) are described by the zero-level set.

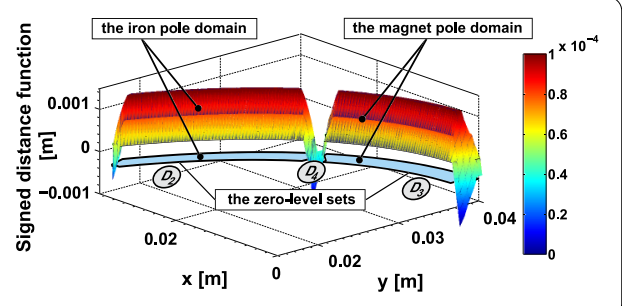

\subsection{Optimization variables and multi-level representation}

In the course of the PM machine design optimization, the placement of iron, permanent magnets and air gaps are subject to variations. To this end, we denote the iron pole domain by $D_{2}$, the magnet pole domain by $D_{3}$ and the air gap region by $D_{4}$, see Figure 4 . Employing the modified multilevel set method (MLSM) [40, 41], we have to trace the two interfaces between different materials (iron/air and permanent magnet/air) by corresponding signed distance functions $\phi_{1}$ and $\phi_{2}$, see also Figure 4. Using the signed distance functions, we can describe the respective domains as:

$$
\begin{array}{ll}
\mathrm{D}_{1}=\left\{\mathbf{x} \in \mathrm{D} \mid \phi_{1}>0 \text { and } \phi_{2}>0\right\}, & \mathrm{D}_{2}=\left\{\mathbf{x} \in \mathrm{D} \mid \phi_{1}>0 \text { and } \phi_{2}<0\right\}, \\
\mathrm{D}_{3}=\left\{\mathbf{x} \in \mathrm{D} \mid \phi_{1}<0 \text { and } \phi_{2}>0\right\}, & \mathrm{D}_{4}=\left\{\mathbf{x} \in \mathrm{D} \mid \phi_{1}<0 \text { and } \phi_{2}<0\right\} .
\end{array}
$$

During optimization the signed distance functions $\phi_{i}(i=1,2)$ are continuously adapted. Their evolution is governed by the following Hamilton-Jacobi-type equation [42]

$$
\frac{\partial \phi_{i}}{\partial t}=-\nabla \phi_{i}(\mathbf{x}, t) \frac{\mathrm{d} \mathbf{x}}{\mathrm{d} t}=V_{n, i}\left|\nabla \phi_{i}\right|
$$

with pseudo-time $t$ and the normal component of the zero-level set velocity $V_{n, i}$.

\subsection{Optimization and uncertainty}

For a robust optimization, that uncertainties of the reluctivities need to be included. We recall our stochastic model for the reluctivity $v(5)$, where $\xi=\left(\xi_{1}, \xi_{2}, \xi_{3}\right)$ denote the stochastic variations in $v$ for Fe, air and PM. In the multi-level set representation, the reluctivity $v$ and the remanent flux density coefficient $b_{r}$ (of the PM-material, see above in (2)) become functions of the random variables $\boldsymbol{\xi}$ and the sign distance functions $\phi=\left(\phi_{1}, \phi_{2}\right)$ :

$$
\begin{aligned}
v(\boldsymbol{\phi}, \boldsymbol{\xi})= & v_{1}\left(\xi_{1}\right) H\left(\phi_{1}\right) H\left(\phi_{2}\right)+v_{2}\left(\xi_{2}\right) H\left(\phi_{1}\right)\left(1-H\left(\phi_{2}\right)\right) \\
& +v_{3}\left(\xi_{3}\right)\left(1-H\left(\phi_{1}\right)\right) H\left(\phi_{2}\right)+v_{4}\left(\xi_{4}\right)\left(1-H\left(\phi_{1}\right)\right)\left(1-H\left(\phi_{2}\right)\right), \\
b_{r}(\boldsymbol{\phi})= & b_{r 1} H\left(\phi_{1}\right) H\left(\phi_{2}\right)+b_{r 2} H\left(\phi_{1}\right)\left(1-H\left(\phi_{2}\right)\right) \\
+ & b_{r 3}\left(1-H\left(\phi_{1}\right)\right) H\left(\phi_{2}\right)+b_{r 4}\left(1-H\left(\phi_{1}\right)\right)\left(1-H\left(\phi_{2}\right)\right)
\end{aligned}
$$

with Heaviside function $H(\cdot)$. Thus the standard level-set-based algorithm $[40,42]$ includes the following steps:

(a) First, after a model initialization, using, e.g., a gradient topological method, the signed distance functions $\phi_{i}, i=1,2$ need to be calculated. In particular, it means that the shapes under colorred the consideration are described by the zero-level 
sets, shown on Figure 4 (without utilizing any additional parametrization function, besides a model discretization).

(b) Based on the knowledge of the zero-level set velocity $V_{n, i}$, modified by area constraints, for which the adjoint variable method or the continuum design sensitivity analysis might be applied, the corrections of the distribution of signed distance functions are calculated and then introduced into the model in every iteration. Here also the distribution of level sets can be modified based on the topological information. Additionally, the Tikhonov regularization or the total validation technique can be used in order to control the complexity/smoothness of the optimized shapes $[6,23]$.

(c) Finally, stops criteria are checked and the optimization process is continued until they will be fulfilled.

\section{Topology optimization under uncertainties}

We have setup a shape optimization problem constrained by the elliptic PDEs (12) with random material variations. Now we need efficient and robust computation strategies.

\subsection{Dual problem}

When a nonlinear magnetostatic problem is considered, a variational formulation of a dual problem needs to be additionally formulated, e.g., [54] and [55]. In a magnetostatic case, it takes the form of

$$
\begin{gathered}
\int_{D_{\mathrm{F}}}\left(v\left(\mathbf{x},|\nabla A|^{2}\right) \nabla \varphi \nabla \zeta\right) \mathrm{d} \mathbf{x}+\int_{D_{\mathrm{F}}}\left(2 \partial_{B} v\left(\mathbf{x},|\nabla A|^{2}\right) \cdot \nabla \varphi \nabla \zeta\right) \mathrm{d} \mathbf{x} \\
\quad=\int_{D_{\mathrm{F}}}\left(\varphi J+v_{\mathrm{PM}} \mathbf{M} \nabla \varphi\right) \mathrm{d} \mathbf{x} \quad \text { for all } \varphi \in V
\end{gathered}
$$

with adjoint variable $\zeta$. A discussion of the existence and uniqueness of solution of (23) can be found in $[45,54]$.

In the steady-state analysis and using a Newton-Raphson algorithm, the adjoint variable $\zeta$ can be computed directly. This is due to the fact that the converged system of the direct problem (4) and the adjoint problem (23) are the same [56]. This technique, the so-called frozen method was successfully applied for calculating the electromagnetic force in the nonlinear magnetostatic system [55] and for providing the on-load CT [57].

\subsection{Robust topology optimization problem}

The minimization of the cogging torque in our 2D magnetostatic case can be equivalently represented as the minimization of the magnetic energy $W_{\mathrm{r}}$ variation [21, 41].

In our context of the shape optimization problem with the PDE constraint (12), the magnetic energy is defined as

$$
W_{\mathrm{r}}\left(\phi_{1}, \phi_{2}, \boldsymbol{\xi}\right)=\int_{\mathrm{D}} \mathbf{B}\left(\phi_{1}, \phi_{2}\right) \mathbf{H}\left(\phi_{1}, \phi_{2}\right) \mathrm{d} \mathbf{x}+\sum_{i=1}^{2} \beta_{i} \operatorname{TV}\left(\phi_{i}\right)
$$

Here $\operatorname{TV}(\cdot)$ denotes the total variation regularization with given coefficient $\beta_{i}$; it is used to control the geometrical complexity of obtained shapes $[6,40]$. 
Now, employing the magnetic energy in the yield function of the minimization process allows us to compute the sensitivity an efficient way [21]. This reads as follows:

$$
\frac{\partial W_{\mathrm{r}}}{\partial \mathbf{p}}=\int_{\gamma}\left(v_{1}-v_{2}\right) \nabla \times \mathbf{A}^{*} \cdot \nabla \times \mathbf{A}^{* *}-\left(\mathbf{M}_{1}-\mathbf{M}_{2}\right) \cdot \nabla \times \mathbf{A}^{* *} \mathrm{~d} \gamma, \quad \text { in D }
$$

with $v_{1}$ and $v_{2}$ as well as $\mathbf{M}_{1}$ and $\mathbf{M}_{2}$ representing reluctivities and remanent flux density for different domains, as well as $\mathbf{A}^{*}:=A$ and $\mathbf{A}^{* *}:=\zeta$ denoting the magnetic vector potential in the primal and dual problem. The vector of parameters $\mathbf{p}$ is defined as follows $\mathbf{p}=\left(\boldsymbol{v}, b_{r}\right)$.

Moreover, (24) is subjected to the constraint (17), where $B_{r}$ is replaced by $B_{r}\left(\phi_{1}, \phi_{2}\right)$. In our optimization procedure, this constraint is realized in an approximative way. It is introduced as two separate area constraints, one for each rotor pole cf. [22, 41, 58]:

$$
\begin{aligned}
& G_{1}(\boldsymbol{\phi})=\left|\mathrm{D}_{2}\right| /\left|\mathrm{D}_{2_{0}}\right|-S_{2}=0, \\
& G_{2}(\boldsymbol{\phi})=\left|\mathrm{D}_{3}\right| /\left|\mathrm{D}_{3_{0}}\right|-S_{3}=0,
\end{aligned}
$$

where $S_{2}$ and $S_{3}$ are prescribed coefficients, which is a kind of standard approach in a robust framework), while $\mathrm{D}_{3_{0}}$ and $\mathrm{D}_{2_{0}}$ represent initial areas of a PM and iron pole, respectively.

Finally, let the random space be sampled at $K+1$ quadrature grid points (using the Stroud formula). Thus, a stochastic multi-objective topology optimization problem is formulated in terms of a robust functional [59], consisting of the expectation and the standard deviation using the WAM [13]:

$$
\begin{aligned}
& \min _{\phi}\left(\mathbb{E}\left[W_{\mathrm{r}}\left(\phi_{1}, \phi_{2}, \boldsymbol{\xi}\right)\right]+\kappa \sqrt{\operatorname{Var}\left[W_{\mathrm{r}}\left(\phi_{1}, \phi_{2}, \boldsymbol{\xi}\right)\right]}\right) \\
& \text { s.t. } \mathbf{K}\left(v^{k}\right) \mathbf{A}^{k}=\mathbf{f}^{k}, \quad k=0, \ldots, K, \\
& \quad\left|\mathrm{D}_{2}\right| \leq S_{2} \cdot\left|\mathrm{D}_{2_{0}}\right| \quad \text { and } \quad\left|\mathrm{D}_{3}\right| \leq S_{3} \cdot\left|\mathrm{D}_{3_{0}}\right|
\end{aligned}
$$

with prescribed parameter $\kappa=3$ (analogously to three-sigma rule of thumb used in statistics and empirical science), and stiffness matrix $\mathbf{K}$.

Here, one can compute the total derivative of the magnetic energy (24) on the basis of the forward analysis, only. That is, the forward model is calculated in the collocation points, while using the models for $v(21), b_{r}$ (22) and the sensitivity (25) as well as the coefficients of the PCE (14) and the moments (15). We remark that a similar approach was used in [60, 61] for the solution of stochastic identification/control problems for constrained PDEs with random input data. However, their type of cost functional was different. It should be also emphasized that in contrary to the work by [22] about the deterministic low ripple torque design, in our paper we deal with the low cogging torque design of the ECPSM machine under uncertainties.

\section{Simulation procedure}

Furthermore, the algorithm for the robust topology optimization has been implemented using the Comsol (COMSOL 3.5a, The COMSOL Inc., Burlington, MA, 2008) and Matlab (MATLAB 7.10, The MathWorks Inc., Natick, MA, 2010) scripts. Thus the Finite Element 
method is used to solve both the weak formulations of primary and dual system, defined by (4) and (23), respectively. The respective triangular mesh consists of 81,016 elements with each second order Lagrange polynomials for the $A$-formulation. As a reluctivity model for iron parts, the soft iron material without losses has been applied and a standard spline interpolation of measured data are used for the nonlinear dependence on $|\nabla A|^{2}$. The computation time (wall clock time) for a fixed position of rotor and stator in our configuration was about $30.31 \mathrm{~s}$. This did involve 163,283 degrees of freedom. Additionally, every rotor pole has been divided into 468 voxels. This applies to PM and iron pole, separately. The UQ analysis has been performed using the software implemented by [62]. The Stroud-5 formula has been used for this purpose. The optimized shapes of rotor poles have been found in the 10th iteration of the optimization process. We have applied the Stroud-3 formula in order to obtain the final results for the UQ analysis of the CT, the back EMF, the electromagnetic torque and the magnetic flux density in the air-gap, respectively.

\section{Numerical results}

The above described procedure has been applied to design the rotor poles of the ECPSM for no-load state, Figure 2, with parameters in Table 1 Subject to optimization is the shape of the iron pole and the PM pole. The initial domains of the respective poles and the corresponding field in the ECPSM machine are represented in Figure 5. This is the starting point for the optimization. In addition, the reluctivities are assumed to be uncertain (5) with maximum deviation of $10 \%^{\mathrm{b}}$ for the respective nominal value. The rotor poles after optimization are depicted in Figure $6 .^{\mathrm{c}}$

Afterwards, to discuss the quality of the design, the CT is computed for two periods of both the initial and the optimized topology. And the interaction of the stator teeth with the rotor poles is investigated. The results for the mean and standard deviation of the CT are depicted in Figure 7. The peak value of the mean value of the CT is reduced about 83\%. In order to investigate the influence the robust optimization on the back EMF and the magnetic flux density in the air-gap under rotor poles, we show the mean and standard deviation calculated for both considered quantities in Figures 8 and 9, respectively. Additionally, for the last quantity we perform a spectral analysis using FFT, which is depicted on Figure 10. Based on this we are able to calculate the total harmonic distortion (THD) ${ }^{\mathrm{d}}$

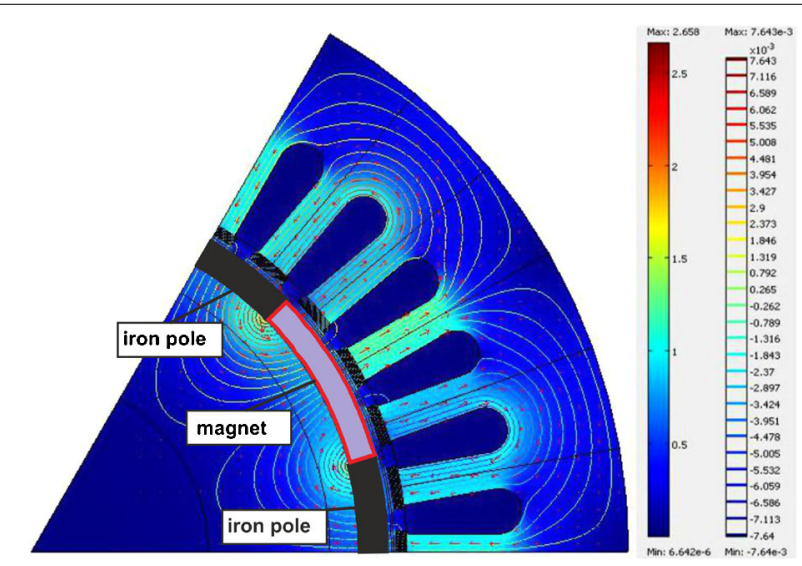

Figure 5 An initial topology of the ECPSM [35]. 


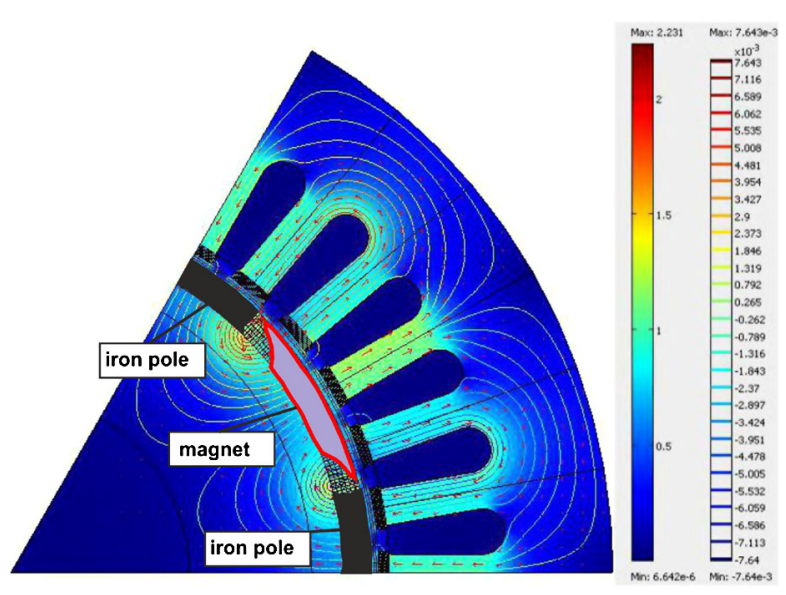

Figure 6 The optimized topology of the ECPSM [35].

Figure 7 Mean and standard deviation for initial and optimized topology of the ECPSM: cogging torque versus mechanical degree.

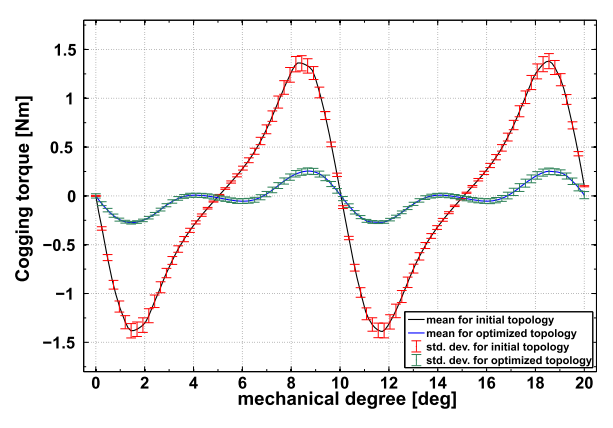

Figure 8 Mean and standard deviation for initial and optimized topology of the ECPSM: magnetic flux density in the air-gap under magnet and iron poles.

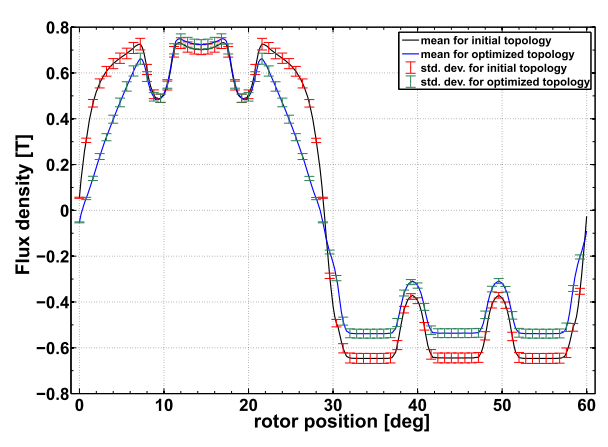

factor that allows for assessing the improvement of waveform of the back EMF, which in our case becomes around 32\%.

Finally, we also computed the electromagnetic torque, shown on Figure 11. It means that we analyzed the machine in an on-load mode with defined excitation currents defined the right side of equation (1). The detailed analysis for the machine before and after the optimization is presented in the form of Table 2. Unfortunately, we have also to report that the root mean square of the flux density in the air-gap, the back EMF and the electromagnetic torque are decreased by around 15\%,10\% and 17\%, respectively. Surely this can be seen as a drawback of the proposed method. On the other hand, robustness is included. 


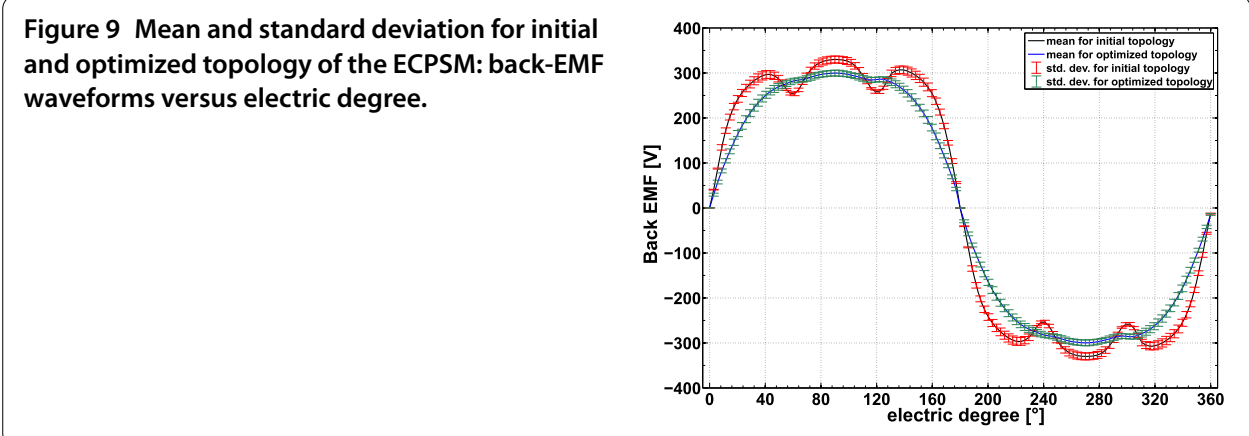

Figure 10 FFT analysis of the back EMF mean for initial and optimized model of ECPSM.

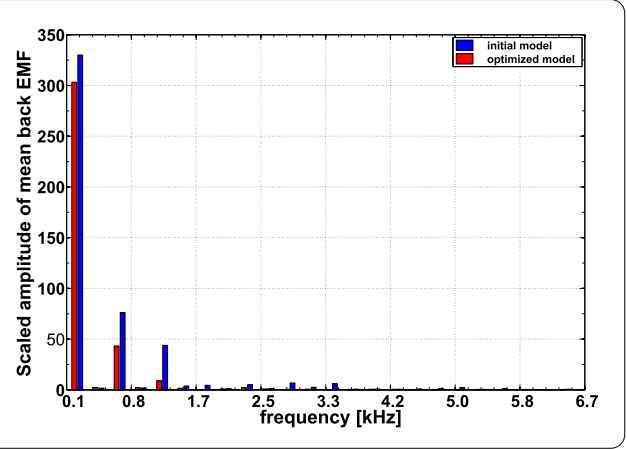

Figure 11 Mean and standard deviation for initial and optimized topology of the ECPSM: electromagnetic torque versus electric degree.

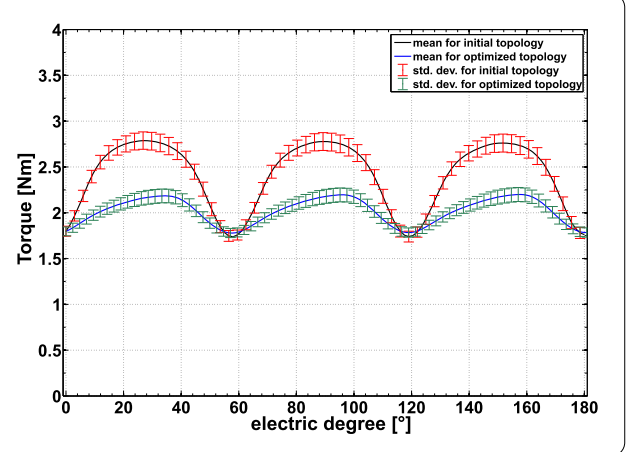

\section{Conclusion}

In this paper we demonstrated how to combine the stochastic collocation method (SCM) with the multi-level set method (MLSM) and how to apply this technique efficiently for the robust topology optimization of a PM synchronous machine. In the end, the shape of rotor poles were optimized also with respect to the level of noise and vibrations. This did result in significant reductions of both the rms of the CT (82\%) and the mean value of the standard deviation (43\%). Thereby we were able to take variations with respect to manufacturing tolerances/imperfections into account by assuming a random field for the reluctivities. As a drawback, we reported a small decrease in the root mean square values of the electromagnetic torque and back EMF. However, it should be noticed that aims of the low cogging torque robust topology optimization (the electric machine in the no-load mode) have been completely fulfilled. Additionally, the waveform has been considerably improved about $32 \%$, while the torque ripple has been reduced around $52 \%$. The detailed analysis presented in Table 2 indicates that further research should be focused on the low 
Table 2 Values of some physical parameters of the ECPSM model before and after optimization

\begin{tabular}{|c|c|c|c|}
\hline Quantity (unit) & $\begin{array}{l}\text { Before } \\
\text { optimization }\end{array}$ & $\begin{array}{l}\text { After } \\
\text { optimization }\end{array}$ & $\begin{array}{l}\text { Decrease/ } \\
\text { increase }\end{array}$ \\
\hline \multicolumn{4}{|c|}{ Expectation of the cogging torque (Nm) } \\
\hline Rectified mean value & 0.072 & 0.012 & $83.70 \% \downarrow$ \\
\hline RMS value & 0.085 & 0.015 & $82.19 \% \downarrow$ \\
\hline Minimal value & -0.139 & -0.027 & $80.21 \% \downarrow$ \\
\hline Maximal value & 0.138 & 0.026 & $80.51 \% \downarrow$ \\
\hline Mean value of standard deviation & 0.004 & 0.002 & $43.19 \% \downarrow$ \\
\hline \multicolumn{4}{|l|}{ Expectation of the back EMF (V) } \\
\hline Rectified mean value & 257.1 & 225.9 & $12.14 \% \downarrow$ \\
\hline RMS value & 268.3 & 240.6 & $10.33 \% \downarrow$ \\
\hline Minimal value & -330.0 & -299.8 & $9.14 \% \downarrow$ \\
\hline Maximal value & 330.0 & 299.8 & $9.14 \% \downarrow$ \\
\hline Mean value of standard deviation & 6.34 & 6.23 & $1.85 \% \downarrow$ \\
\hline \multicolumn{4}{|c|}{ Expectation of the air-gap magnetic flux density $(\mathrm{T})$} \\
\hline Rectified mean value & 0.575 & 0.471 & $18.08 \% \downarrow$ \\
\hline RMS value & 0.592 & 0.502 & $15.11 \% \downarrow$ \\
\hline Minimal value & -0.647 & -0.539 & $16.73 \% \downarrow$ \\
\hline Maximal value & 0.733 & 0.749 & $2.29 \% \uparrow$ \\
\hline Mean value of standard deviation & 0.019 & 0.017 & $12.29 \% \downarrow$ \\
\hline \multicolumn{4}{|c|}{ Expectation of the electromagnetic torque (Nm) } \\
\hline Rectified mean value & 2.407 & 2.014 & $16.31 \% \downarrow$ \\
\hline RMS value & 2.433 & 2.019 & $17.02 \% \downarrow$ \\
\hline Minimal value & 1.738 & 1.779 & $2.31 \% \uparrow$ \\
\hline Maximal value & 2.788 & 2.200 & $21.11 \% \downarrow$ \\
\hline Mean value of standard deviation & 0.09 & 0.063 & $20.33 \% \downarrow$ \\
\hline \multicolumn{4}{|l|}{ Expectation of others quantities } \\
\hline Ripple torque (\%) & 43.62 & 20.90 & $52.10 \% \downarrow$ \\
\hline THD of the back EMF (VN) & 0.732 & 0.498 & $31.93 \% \downarrow$ \\
\hline Mass of iron pole (g) & 15.95 & 14.94 & $6.32 \% \downarrow$ \\
\hline Mass of PM pole (g) & 15.95 & 12.19 & $23.56 \% \downarrow$ \\
\hline
\end{tabular}

ripple torque robust design in the on-load state (with excitation currents included). Then, the robust optimization of the electric machine could be performed when taking both the ripple torque and the average electromagnetic torque into account. This is considered as a further direction of our investigation. This work also highlights the effectiveness of the proposed methodology.

Competing interests

The authors declare that they have no competing interests.

\section{Authors' contributions}

All authors contributed to the writing of the final version of this paper. However, a main concept of the proposed method was developed by PP. All authors read and approved the final manuscript.

\section{Author details}

${ }^{1}$ Chair of Applied Mathematics and Numerical Analysis, Bergische Universität Wuppertal, Gaußstraße 20, Wuppertal, 42119, Germany. ${ }^{2}$ Institute for Mathematics and Computer Science, Ernst-Moritz-Arndt-Universität Greifswald, Walther-Rathenau-Straße 47, Greifswald, 17489, Germany. ${ }^{3}$ Department of Electrotechnology and Diagnostic, West Pomeranian University of Technology, Piastów 17, Szczecin, 70-310, Poland.

\section{Acknowledgements}

The project nanoCOPS (Nanoelectronic COupled Problems Solutions) is supported by the European Union in the FP7-ICT-2013-11 Program under the grant agreement number 619166 and the SIMUROM project is supported by the German Federal Ministry of Education and Research (05M13PXB). The authors would also like to thank Dr. Piotr Paplicki, West Pomeranian University of Technology in Szczecin, for his help in the final simulations and Professor Ryszard Pałka, West Pomeranian University of Technology in Szczecin, for the opportunity to use the ECPSM model in our research. We are also thankful to Kai Gausling, MSc., University of Wuppertal, for using his software for the uncertainty quantification. 


\section{Endnotes}

a For an orthogonal system of basis polynomials a normalization can be done straightforward, e.g., [33].

b Due to the used Stroud quadrature formulas [51], the same distribution had to be assumed with a relatively high variance based on [38] for the reluctivity of a PM.

C A similar PM machine was also the topic of the scientific project 'The Electrically Controlled Permanent Magnet Excited Synchronous Machine (ECPSM) with application to electro-mobiles' under the Grant No. N510 508040, founded by Polish Government. There the topology was deterministically optimized.

$\mathrm{d}$ The THD is defined as: THD $=\sqrt{\frac{V_{2}^{2}+v_{3}^{2}+V_{4}^{2}+\cdots+V_{n}^{2}}{V_{1}^{2}}}$, where $V_{k}$ is the root mean square voltage of the $k$ th harmonic and $k=1$ denotes the fundamental frequency.

Received: 2 March 2016 Accepted: 31 October 2016 Published online: 08 November 2016

\section{References}

1. Gieras JF, Wing M. Permanent magnet motor technology. New York: Wiley; 2008.

2. Paplicki P, Wardach M, Bonislawski M, Pałka R. Simulation and experimental results of hybrid electric machine with a novel flux control strategy. Arch Electr Eng. 2015;64(1):37-51.

3. Di Barba P, Bonisławski M, Pałka R, Paplicki P, Wardach M. Design of hybrid excited synchronous machine for electrical vehicles. IEEE Trans Magn. 2015;51(3):8107206.

4. Brasel M. A gain-scheduled multivariable LQR controller for hybrid excitation synchronous machine. In: 20th international conference on methods and models in automation and robotics MMAR 2015. 24-27 Aug. 2015, Poland; 2015. p. 655-8.

5. Makni Z, Besbes M, Marchand C. Multiphysics design methodology of permanent-magnet synchronous motors. IEEE Trans Veh Technol. 2007;56(4):1524-30.

6. Putek P, Paplicki P, Pałka R. Low cogging torque design of permanent magnet machine using modified multi-level set method with total variation regularization. IEEE Trans Magn. 2014;50(2):657-60.

7. Paplicki P. Design optimization of the electrically controlled permanent magnet excited synchronous machine to improve flux control range. Electron Electrotechn. 2014;20(4):17-22.

8. Putek P, Paplicki P, Slodička M, Pałka R, Van Keer R. Application of topological gradient and continuum sensitivity analysis to the multi-objective design optimization of a permanent-magnet excited synchronous machine. Electr Rev. 2012;88(7a):256-60.

9. Bianchi N, Bolognani S. Design techniques for reducing the cogging torque in surface-mounted PM motors. IEEE Trans Ind Appl. 2002;38(5):1259-65.

10. Chen S, Namuduri C, Mir S. Controller-induced parasitic torque ripples in a PM synchronous motor. IEEE Trans Ind Appl. 2002;38(5):1273-81.

11. Islam MS, Islam R, Sebastian T. Experimental verification of design techniques of permanent-magnet synchronous motors for low-torque-ripple applications. IEEE Trans Ind Appl. 2011;47(1):88-95.

12. Putek P, Paplicki P, Pulch R, ter Maten EJW, Günther M, Pałka R. Multi-objective topology optimization of a permanent magnet machine to reduce electromagnetic losses. Int J Appl Electromagn Mech. 2016. Accepted.

13. Marler RT, Arora JS. Survey of multi-objective optimization methods for engineering. Struct Multidiscip Optim. 2004:26(6):369-95.

14. Di Barba P. Multi-objective shape design in electricity and magnetism. Berlin: Springer; 2010.

15. De Tommasi L, Beelen TGJ, Sevat MF, Rommes J, ter Maten EJW. Multi-objective optimization of RF circuit blocks via surrogate models and NBI and SPEA2 methods. In: Günther M, Bartel A, Brunk M, Schöps S, Striebel M, editors. Progress in industrial mathematics at ECMI 2010. Mathematics in industry. vol. 17. Berlin: Springer; 2012. p. $195-201$.

16. Batista J, Zuliani Q, Weiss-Cohen M, de Souza-Batista L, Gadelha-Guimarães F. Multi-objective topology optimization with ant colony optimization and genetic algorithms. Comput-Aided Des Appl. 2015;12(6):674-82.

17. Moehle N, Boyd S. Optimal current waveforms for brushless permanent magnet motors. Int J Control. 2015;88(7):1389-99.

18. Zhu ZQ, Howe D. Influence of design parameters on cogging torque in permanent magnet machines. IEEE Trans Energy Convers. 2000;15(4):407-12.

19. Hwang CC, John SB, Wu SS. Reduction of cogging torque in spindle motors for CD-ROM drive. IEEE Trans Magn. 1998;34(2):468-70

20. Kwack J, Min S, Hong JP. Optimal stator design of interior permanent magnet motor to reduce torque ripple using the level set method. IEEE Trans Magn. 2010;46(6):2108-11.

21. Kim D, Sykulski J, Lowther D. The implications of the use of composite materials in electromagnetic device topology and shape optimization. IEEE Trans Magn. 2009:45:1154-6.

22. Lim S, Min S, Hong JP. Low torque ripple rotor design of the interior permanent magnet motor using the multi-phase level-set and phase-field concept. IEEE Trans Magn. 2012;48(2):907-9.

23. Yamada T, Izui K, Nishiwaki S, Takezawa A. A topology optimization method based on the level set method incorporating a fictitious interface energy. Comput Methods Appl Mech Eng. 2010;199(45-48):2876-91.

24. Putek P. Mitigation of the cogging torque and loss minimization in a permanent magnet machine using shape and topology optimization. Eng Comput. 2016;33(3):831-54.

25. Li JT, Liu ZJ, Jabbar MA, Gao XK. Design optimization for cogging torque minimization using response surface methodology. IEEE Trans Magn. 2004;40(2):1176-80.

26. Islam MS, Islam R, Sebastian T, Chandy A, Ozsoylu SA. Cogging torque minimization in PM motors using robust design approach. IEEE Trans Magn. 2011;47(4):1661-9.

27. Kim N-K, Kim D-H, Kim D-W, Kim H-G, Lowther DA, Sykulski JK. Robust optimization utilizing the second-order design sensitivity information. IEEE Trans Magn. 2010;46(8):3117-20.

28. Abdallh A, Crevecoeur G, Dupré L. Impact reduction of the uncertain geometrical parameters on magnetic material identification of an El electromagnetic inductor using an adaptive inverse algorithm. J Magn Magn Mater. 2012;324(7):1353-9. 
29. Römer U, Schöps S, Weiland T. Approximation of moments for the nonlinear magnetoquasistatic problem with material uncertainties. IEEE Trans Magn. 2014;50(2):7010204.

30. Babučka I, Nobile F, Tempone R. A stochastic collocation method for elliptic partial differential equations with random input data. SIAM J Numer Anal. 2010;52(2):317-55.

31. Babučka I, Nobile F, Tempone R. Worst-case scenario for elliptic problems with uncertainty. Numer Math. 2005:101(2):185-219.

32. Xiu D. Efficient collocational approach for parametric uncertainty analysis. Commun Comput Phys. 2007;2(2):293-309.

33. Xiu D. Numerical methods for stochastic computations: a spectral method approach. Princeton: Princeton University Press; 2010.

34. Putek $P$, Meuris $P$, Pulch $R$, ter Maten EJW, Schoenmaker W, Günther M. Uncertainty quantification for a robust topology optimization of power transistor devices. IEEE Trans Magn. 2016;52(3):1700104.

35. Putek P, Gausling K, Bartel A, Gawrylczyk KM, ter Maten EJW, Pulch R, Günther M. Robust topology optimization of a permanent magnet synchronous machine using level set and stochastic collocation methods. In: Bartel A, Clemens M, Günther M, ter Maten EJW, editors. Scientific computing in electrical engineering SCEE 2014. Mathematics in industry. vol. 23. Berlin: Springer; 2016. p. 233-42.

36. ter Maten EJW, Putek PA, Günther M, Pulch R, Tischendorf C, Strohm C, Schoenmaker W, Meuris P, De Smedt B, Benner P, Feng L, Banagaaya N, Yue Y, Janssen R, Dohmen JJ, Tasić B, Deleu F, Gillon R, Wieers A, Brachtendorf H-G, Bittner K, Kratochvil T, Petřzela J, Sotner R, Götthans T, Dřinovský J, Schöps S, Duque Guerra DJ, Casper T, De Gersem H, Römer U, Reynier P, Barroul P, Masliah D, Rousseau B. Nanoelectronic COupled problems solutions - nanoCOPS: modelling, multirate, model order reduction, uncertainty quantification, fast fault simulation. J Math Ind. 2016;7:2.

37. Sergeant $P, C$ Crevecoeur $G$, Dupré L, van den Bossche A. Characterization and optimization of a permanent magnet synchronous machine. Compel. 2008;28(2):272-84

38. Rovers JMM, Jansen JW, Lomonova EA. Modeling of relative permeability of permanent magnet material using magnetic surface charges. IEEE Trans Magn. 2013;49(6):2913-9.

39. Bartel A, De Gersem H, Hülsmann T, Römer U, Schöps S, Weiland T. Quantification of uncertainty in the field quality of magnets originating from material measurements. IEEE Trans Magn. 2013;49(5):2367-70.

40. Vese LA, Chan TF. A multiphase level set framework for image segmentation using the Mumford and Shah model. Int J Comput Vis. 2002;50(3):271-93.

41. Putek P, Paplicki P, Pałka R. Topology optimization of rotor poles in a permanent-magnet machine using level set method and continuum design sensitivity analysis. Compel. 2014;33(6):711-28.

42. Osher SJ, Sethian JA. Fronts propagating with curvature dependent speed: algorithms based on Hamilton-Jacobi formulations. J Comput Phys. 1988;79(1):12-49.

43. Verma S, Balan A. Experimental investigations on the stators of electrical machines in relation to vibration and noise problems. IEE Proc, Electr Power Appl. 1998;145(5):15-8.

44. May H, Pałka R, Paplicki P, Szkolny S, Canders WR. Modified concept of permanent magnet excited synchronous machines with improved high-speed features. Arch Elektrotech. 2011;60(4):531-40.

45. Bachinger $F$, Langer $U$, Schöberl J. Numerical analysis of nonlinear multiharmonic eddy current problems. Numer Math. 2005;100(4):593-616.

46. Wiener N. The homogeneous chaos. Am J Math. 1938;60(4):897-936.

47. Xiu D, Karniadakis GE. The Wiener-Askey polynomial chaos for stochastic differential equations. SIAM I Sci Comput. 2002;24(2):619-44

48. Ernst OG, Mugler A, Starkloff HJ, Ullmann E. On the convergence of generalized polynomial chaos expansions. ESAIM: Math Model Numer Anal. 2012;46(2):317-39.

49. Pulch R. Stochastic collocation and stochastic Galerkin methods for linear differential algebraic equations. J Comput Appl Math. 2014;262:281-91.

50. Xiu D, Hesthaven JS. High-order collocation methods for differential equations with random inputs. SIAM J SCi Comput. 2005;27(3):1118-39

51. Stroud AH. Some fifth degree integration formulas for symmetric regions. Math Comput. 1966;20(93):90-7.

52. Lee JH, Kim DH, Park IH. Minimization of higher back-EMF harmonics in permanent magnet motor using shape design sensitivity with B-spline parametrization. IEEE Trans Magn. 2003;39(3):1269-72.

53. Putek P, Paplicki P, Slodička M, Pałka R. Minimization of cogging torque in permanent magnet machines using the topological gradient and adjoint sensitivity in multi-objective design. Int J Appl Electromagn Mech. 2012;39(1-4):933-40.

54. Cimrak I. Material and shape derivative method for quasi-linear elliptic systems with applications in inverse electromagnetic interface problems. SIAM J Numer Anal. 2012;50(3):1086-110.

55. Kim D, Lowther D, Sykulski J. Efficient force calculation based on continuum sensitivity analysis. IEEE Trans Magn. 2005;41(5):1404-7.

56. Park IH, Lee HB, Kwak IG, Hahn SY. Design sensitivity analysis for nonlinear magnetostatic problems using finite element method. IEEE Trans Magn. 1992;28(2):1533-6.

57. Chu WQ, Zhu ZQ. On-load cogging torque calculation in permanent magnet machines. IEEE Trans Magn. 2013:49(6):2982-9.

58. Putek P, Crevecoeur G, Slodička M, Van Keer R, Van de Wiele B, Dupré L. Space mapping methodology for defect recognition in eddy current testing - type NDT. Compel. 2012;31:881-94.

59. Yao W, Chen X, Luo W, van Tooren M, Guo J. Review of uncertainty-based multidisciplinary design optimization methods for aerospace vehicles. Prog Aerosp Sci. 2011;47(6):450-79.

60. Teckentrup AL, Jantsch P, Webster CG, Gunzburger M. A multilevel stochastic collocation method for partial differential equations with random input data. arXiv:1404.2647 (2014).

61. Tiesler H, Kirby RM, Xiu D, Preusser T. Stochastic collocation for optimal control problems with stochastic PDE constraints. SIAM J Numer Anal. 2012;50(5):2659-82.

62. Gausling K, Bartel A. First results for uncertainty quantification in co-simulation of coupled electrical circuits. In: Bartel A, Clemens M, Günther M, ter Maten EJW, editors. Scientific computing in electrical engineering SCEE 2014. Mathematics in industry. vol. 23. Berlin: Springer; 2016. p. 233-42. 